\title{
Tight-Binding Molecular-Dynamics Study of Amorphous Carbon
}

\author{
C. Z. Wang, K. M. Ho, and C. T. Chan \\ Ames Laboratory and Department of Physics and Microelectronics Research Center, Iowa State University, Ames, Iowa 50011
}

(Received 18 May 1992; revised manuscript received 13 November 1992)

The structural and electronic properties of amorphous carbon are studied with tight-binding molecular-dynamics simulations. An amorphous carbon structure with 216 atoms obtained from our simulation gives a structure factor $S(Q)$ in very good agreement with the results of neutron scattering from a sputtered $a-C$ sample. We found that the amorphous structure consists of graphitelike fragments embedded in a matrix of both twofold and fourfold coordinated atoms. We also found a small pseudogap in the electronic density of states at the Fermi level.

PACS numbers: 61.43.Dq, 34.20.Cf, 71.25.Mg

Amorphous carbon $(a-C)$ attracts continuing attention because of its importance in technological applications as well as its fascination from a scientific point of view. The ability of carbon to form strong chemical bonds under a variety of coordination numbers (from 2 to 4 ) endows the noncrystalline phase of carbon with many unique properties, and at the same time makes the system quite complex. Experimentally, most amorphous carbon samples are prepared by evaporation or sputtering of graphite or by mass-selected ion beam deposition (MSIB) techniques [1]. While the $a-C$ samples produced by the evaporation or sputtering techniques are $s p^{2}$ rich, the carbon films obtained by the MSIB are found to be dominated by diamondlike $s p^{3}$ bonding [1]. Despite the considerable theoretical and experimental efforts [1-11] that have been devoted to the study of this fascinating material, the understanding of its microscopic structure and physical properties is still far from complete. Even the most basic information such as the ratios of twofold, threefold, and fourfold sites are still subject to controversy.

In this paper, we report a study of the structural and electronic properties of $a-\mathrm{C}$ using a novel molecular-dynamics scheme which incorporates the electronic structure into molecular dynamics (MD) through an empirical tight-binding Hamiltonian. We focus here on the lower density regime; therefore, the $a-C$ structures presented in this paper should be comparable to those obtained experimentally using evaporation or sputtering preparation techniques (e.g., Ref. [7]). Under a density of 2.20 $\mathrm{g} / \mathrm{cm}^{3}$, we obtained an $a-C$ network which gives a structure factor $S(Q)$ in very good agreement with neutron scattering measurements recently obtained by $\mathrm{Li}$ and Lannin [7] on a sputtered $a$-C sample. The electronic density of states of the $a-C$ network also exhibits a clear deep minimum at the Fermi level, similar to that observed in experiments [1]. We found that amorphous structure in this density regime consists of graphitelike fragments embedded in a matrix of both twofold and fourfold coordinated atoms. Our observation of twofold atoms in the $a-\mathrm{C}$ network provides new insights into the properties of $a-\mathrm{C}$.

In our tight-binding molecular-dynamics (TBMD) scheme, the system is described by a Hamiltonian of the form

$$
\begin{aligned}
H\left(\left\{\mathbf{r}_{i}\right\}\right)= & \sum_{i} \frac{\mathbf{P}_{i}^{2}}{2 m}+\sum_{n}^{\text {occupied }}\left\langle\psi_{n}\left|H_{\mathrm{TB}}\left(\left\{\mathbf{r}_{i}\right\}\right)\right| \psi_{n}\right\rangle \\
& +E_{\mathrm{rep}}\left(\left\{\mathbf{r}_{i}\right\}\right),
\end{aligned}
$$

where $\left\{\mathbf{r}_{i}\right\}$ denote the positions of the atoms ( $i$ $=1,2, \ldots, N)$, and $\mathbf{P}_{i}$ denotes the momentum of the $i$ th atom. The first term in (1) is the kinetic energy of the ions, the second term is the electronic band-structure energy calculated from a parametrized tight-binding Hamiltonian $H_{\mathrm{TB}}\left(\left\{\mathbf{r}_{i}\right\}\right)$, and the third term is a short-ranged repulsive energy. In this scheme, the quantum mechanical nature of the covalent bonding among the carbon atoms is taken into account explicitly in the electronic structure which is calculated for each of the atomic configurations in the MD simulations. Details of the tight-binding model are described in Ref. [12]. The accuracy and transferability of this model have been well tested: It describes accurately the properties of crystalline carbon structures with various coordination numbers including the linear carbon chain, graphite, and diamond structures, as well as more complex systems such as liquid carbon and the structures of carbon clusters with sizes ranging from 5 to 100 atoms [12-14].

The amorphous structures are generated in our simulations by quenching from the liquid phase. The simulations are performed with 216 atoms in a cubic cell with periodic boundary conditions. The $\Gamma$ point is used for the electronic calculations. The time step used in our simulations is $1.05 \times 10^{-15} \mathrm{~s}$. In order to understand the effects of density on the microscopic structure of the amorphous phase, we perform simulations at three different densities of $2.20,2.44$, and $2.69 \mathrm{~g} / \mathrm{cm}^{3}$. The initial configurations are liquid structures at $5000 \mathrm{~K}$ for each given density [15]. The volumes and shapes of the MD cells are fixed during the quenching process. The stochastic temperature control method [16] is used to control the temperature, with a quench rate of about $500 \mathrm{~K} / \mathrm{ps}$. The structural and electronic properties of the amorphous structure are analyzed at $T=700 \mathrm{~K}$. This temperature is 
comparable to the experimental condition of room temperature if one includes the effect of zero point motion of the carbon atoms. After 2000 MD steps (corresponding to $2 \mathrm{ps}$ ) of thermalization at $T=700 \mathrm{~K}$, another 2000 MD steps without temperature control are used to perform the statistical average for the system.

Figure 1 shows the simulation results for the structure factor $S(Q)$ and pair correlation function $g(r)$ of our $a-\mathrm{C}$ structure with a density of $2.20 \mathrm{~g} / \mathrm{cm}^{3}$. Our results agree well with the neutron scattering data (dashed line) recently measured by Li and Lannin [7]. Detailed structural analysis shows that this $a$-C sample contains $80.6 \%$ threefold sites, $7.4 \%$ fourfold sites, and $12 \%$ of twofold sites. The average coordination number is 2.96 . The percentage of fourfold sites found in our simulation is in good agreement with the value of $(6.5 \pm 1.5 \%)$ estimated by a nuclear magnetic resonance (NMR) study [10] on the same $a-\mathrm{C}$ sample used in the neutron scattering measurement [7]. However, the presence of twofold coordinated atoms was not considered in the data analysis of Ref. [7]. In view of the good agreement between experiment and simulation for the structure factor $S(Q)$ and the percentage of fourfold sites, it would be interesting to
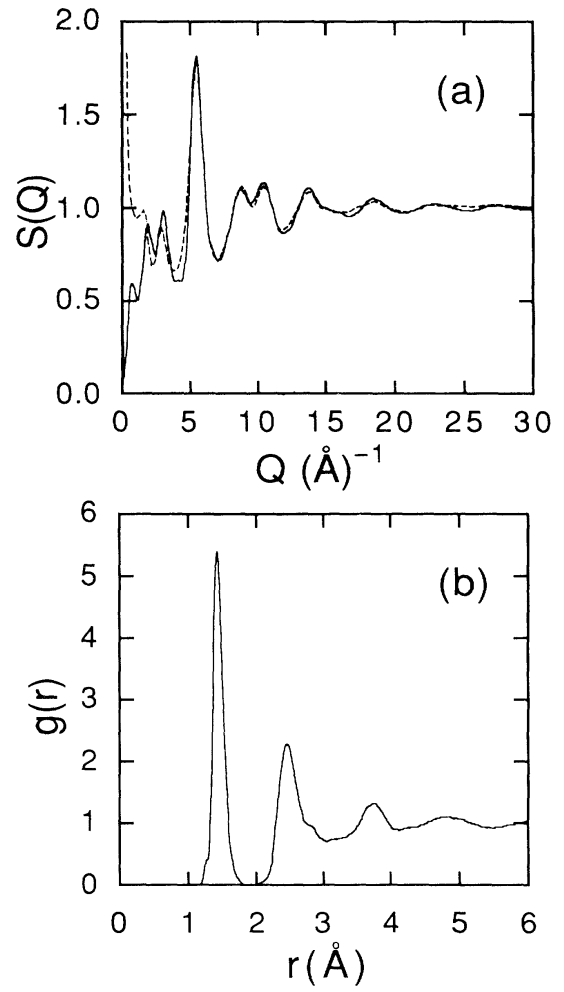

FIG. 1. (a) TBMD result of structure factor $S(Q)$ (solid line) is compared to that of Ref. [7] (dashed line). The density of the TBMD $a-C$ sample is $2.2 \mathrm{~g} / \mathrm{cm}^{3}$, while that of the $a-C$ film used in Ref. [7] was estimated to be between 2.0 and 2.44 $\mathrm{g} / \mathrm{cm}^{3}$. (b) TBMD result of pair correlation function $g(r)$ for the $a$-C sample of $2.2 \mathrm{~g} / \mathrm{cm}^{3}$. reanalyze the experimental data $[7,10]$, including the effects of twofold coordination. In fact, an extended $\mathrm{x}$ ray absorption fine-structure spectroscopy (EXAFS) experiment by Comelli et al. [11] suggested that twofold configurations are necessary to explain the EXAFS data from evaporated and sputtered $a$-C samples.

In Fig. 2, the atomic arrangement of the amorphous carbon obtained by the present simulation with a density equal to $2.20 \mathrm{~g} / \mathrm{cm}^{3}$ is plotted. We found that the threefold coordinated atoms in this amorphous structure form several clusters embedded in the matrix of both twofold and fourfold atoms. Side views of Fig. 2 (not shown in this paper) and a quantitative ring statistics analysis [17] show that these clusters are graphitelike sheets of sizes about $10 \AA \times 10 \AA$, containing mostly six- and some fiveand seven-membered rings. Most of the twofold as well as the fourfold coordinated atoms serve as bridges to hold together the graphitelike clusters. This unique feature is also observed in the other two higher-density samples. However, as the density increases (from 2.20 to 2.69 $\mathrm{g} / \mathrm{cm}^{3}$ ), the number of fourfold sites increases from $7.4 \%$ to $20 \%$, and twofold sites decrease from $12 \%$ to $6.5 \%$ (see Table I). At the same time, the graphitelike clusters shrink in size while the "bridge" regions expand and are increasingly dominated by fourfold atoms which also tend to form clusters. This picture of graphitelike clusters embedded in a random matrix of disordered twofold and fourfold configurations is similar to the models proposed

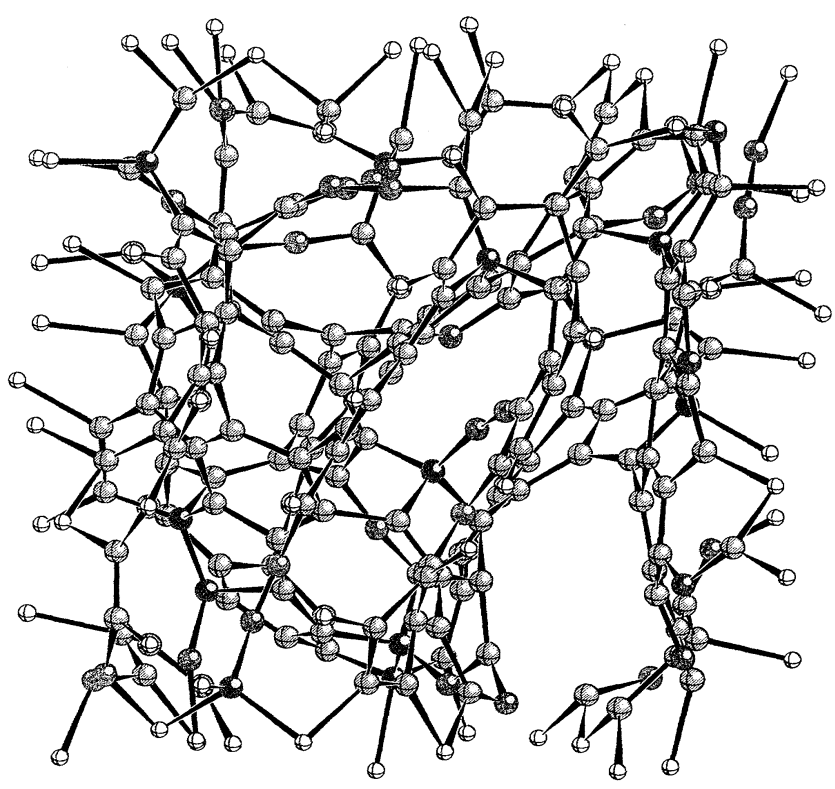

FIG. 2. Microscopic structure of the TBMD-generated $a-C$ network. The density of the sample is $2.2 \mathrm{~g} / \mathrm{cm}^{3}$. Various coordinated atoms are colored differently (twofold: green; threefold: black and white; fourfold: red). Smaller balls represent the carbon atoms outside the central cell (periodic boundary conditions are used). 
TABLE I. The ratios of various coordinated atoms of amorphous carbon obtained by TBMD with 216 atoms. $n_{c}$ is the average coordination number.

\begin{tabular}{ccccc}
\hline \hline $\begin{array}{c}\text { Density } \\
\left(\mathrm{g} / \mathrm{cm}^{3}\right)\end{array}$ & $\begin{array}{c}\text { Twofold } \\
(\%)\end{array}$ & $\begin{array}{c}\text { Threefold } \\
(\%)\end{array}$ & $\begin{array}{c}\text { Fourfold } \\
(\%)\end{array}$ & $n_{c}$ \\
\hline 2.20 & 12.0 & 80.6 & 7.4 & 2.96 \\
2.44 & 5.8 & 80.0 & 14.2 & 3.08 \\
2.69 & 6.5 & 71.0 & 22.5 & 3.16 \\
\hline
\end{tabular}

several years ago by Robertson and O'Reilly based on electronic structure analysis [18] and by Comelli, Stohr, Robinson, and Jark based on EXAFS results [11]. Nevertheless, these models considered either only fourfold coordinated [18] configurations or only twofold coordinated [11] configurations for the random matrix while our simulation showed that both configurations are present with ratios sensitive to the density of the sample.

Although many features of the $a$-C structure obtained from our simulation agree well with that obtained by the $a b$ initio molecular-dynamics simulation of Galli et al. [2], there are some subtle differences. Our simulation for a density of $2.20 \mathrm{~g} / \mathrm{cm}^{3}$ produces $80.6 \%$ threefold sites, $7.4 \%$ fourfold sites, and $12 \%$ twofold sites, whereas the $a b$ initio molecular-dynamics simulation of Galli et al. [2] for a density of $2.0 \mathrm{~g} / \mathrm{cm}^{3}$ gives $85 \%$ threefold sites, $15 \%$ fourfold sites, and no twofold sites at all. The existence of twofold atoms in our simulation should be contrasted to the absence of twofold atoms in the ab initio MD result of Ref. [2]. Moreover, the percentage of fourfold atoms from the $a b$ initio MD seems a bit too high compared with our result as well as the experimental data of Ref. [10]. We note that the energy of linear carbon chain (twofold) with respect to that of diamond (fourfold) and graphite (threefold) from our tight-binding model is $\mathbf{1 . 2 0}$ and $1.22 \mathrm{eV} /$ atom, respectively, which are in excellent agreement with the values of 1.22 and $1.24 \mathrm{eV} /$ atom obtained from converged first-principles local-densityapproximation (LDA) calculations [19,20]. Therefore, the twofold atoms in the $a-\mathrm{C}$ network obtained from our simulation are not an artifact of our potential model. Zhu, Chan, and Ho [20] have performed a LDA planewave calculation [using the same Bachelet-HamannSchlüter (BHS) potential as quoted in Ref. [2]] and found that the relative energies of the linear chain, diamond, and graphite are highly sensitive to the number of plane waves used in the calculation. They found that the energy difference between the linear chain and the diamond structure is $1.72 \mathrm{eV} /$ atom for using the basis set with a kinetic energy cutoff of $20 \mathrm{Ry}$, but is $1.26 \mathrm{eV} /$ atom with a cutoff of $35 \mathrm{Ry}$. They also found that at a $20 \mathrm{Ry}$ cutoff the energy of the graphite structure is higher than that of the diamond structure by about $0.16 \mathrm{eV} / \mathrm{atom}$. The simulation in Ref. [2] was initially performed with a $20 \mathrm{Ry}$ plane-wave cutoff, which is grossly inadequate for the BHS pseudopotential, and, in particular, it overestimates the linear chain energy by about $0.50 \mathrm{eV} / \mathrm{atom}$ $(\sim 5000 \mathrm{~K})$ with respect to diamond. Under such conditions, it is natural that the concentration of twofold carbon atoms is seriously underestimated and that of fourfold carbon atoms is overestimated. The authors of Ref. [2] mentioned that they reannealed the $a$-C structure obtained with the small basis set by using a basis set with a 32 Ry cutoff, and obtained very similar distributions of twofold, threefold, and fourfold atoms. With such a great difference in the relative energies of various coordinated structures, it is quite unlikely that the results for 20 and 32 Ry cutoff simulations can be unchanged. We take this as an indication that the reannealing at $32 \mathrm{Ry}$ in Ref. [2] does not have enough time and/or a high enough temperature for the $a-\mathrm{C}$ network to get out of the metastable configurations (which overemphasizes fourfold $\mathrm{C}$ atoms and underestimates the number of twofold carbon atoms) "frozen in" by the quenching performed at small cutoff.

Finally', we wish to discuss another interesting aspect: the electronic structure of $a-\mathrm{C}$. Experimentally, it has been shown that most evaporated $a-\mathrm{C}$ films exhibit a small optical gap $(\sim 0.5 \mathrm{eV})$. Whether a theoretical structural model of $a-\mathrm{C}$ can produce this optical gap is usually considered as an important criterion for judging the reality of the model. Robertson and O'Reilly [18] performed tight-binding calculations for the structural models proposed by Beeman et al. [21] and found no optical gaps in these models. Robertson and O'Reilly [18] argued that in order to open a gap in the $\pi$ band, it is necessary to have medium-ranged order in the form of graphitelike clusters of dimensions of about $15 \AA$, in the amorphous structure. Figure 3 is a plot of the electronic density of states (DOS) obtained from our simulation at $700 \mathrm{~K}$. The data have been averaged over 2000 configurations. We found that the valence band DOS obtained from our simulation is very similar to the photoemission spectra of Wesner et al. [22]. We found a deep

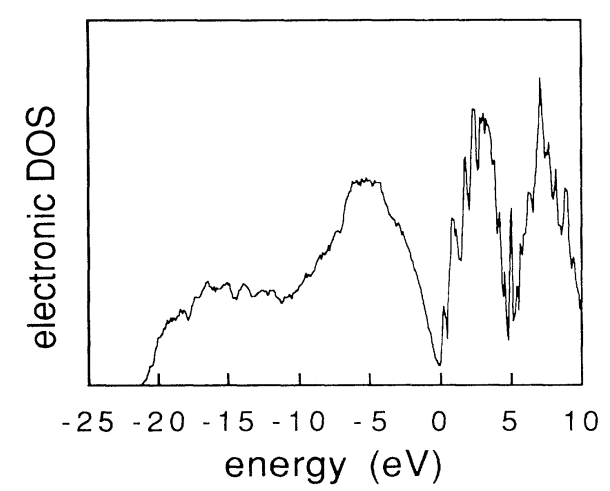

FIG. 3. Electronic density of states of the TBMD-generated $a$-C sample with a density of $2.2 \mathrm{~g} / \mathrm{cm}^{3}$. Note a small pseudogap at the Fermi level $(E=5)$. 
minimum in the DOS near the Fermi level, similar to the experimental measurement [1] and $a b$ initio MD result [2]. It is interesting to note that the electronic DOS obtained from our three samples of different densities are similar, despite the fact that the dimensions of the graphitelike clusters have been reduced by a factor of 2 when the density increases from 2.20 to $2.69 \mathrm{~g} / \mathrm{cm}^{3}$. The width of the pseudogap decreases slightly as the density of the $a-\mathrm{C}$ sample is increased.

In summary, we have shown that tight-binding molecular dynamics can be used for realistic studies of the structural and electronic properties of amorphous carbon. Our results support the proposals of Comelli et al. [11] and Robertson and O'Reilly [18] that sputtered or evaporated $a-\mathrm{C}$ consists of graphitelike regions embedded in a random matrix of disordered twofold [11] and fourfold [18] coordinated atoms. The existence of twofold atoms in the $a-\mathrm{C}$ network in this density regime has been overlooked for many years. We hope that our new results will stimulate further experimental as well as theoretical studies.

We would like to thank Professor R. Martin and Dr. G. Galli for useful discussions and thank Dr. Z. Zhu for performing the LDA plane-wave calculations and Dr. J. Tersoff for useful discussion on the ring statistics analysis. Ames Laboratory is operated for the U.S. Department of Energy by Iowa State University under Contract No. W-7405-Eng-82. This work was supported by the Director for Energy Research, Office of Basic Energy Science, including a grant of computer time at the NERSC at Livermore and by NSF Grant No. DMR8819379 .

[1] For a review, see J. Robertson, Adv. Phys. 35, 317 (1986); and in Diamond and Diamond-like Films and Coatings, edited by R. Clausing et al., NATO Advanced Study Institutes Ser. B, Vol. 266 (Plenum, New York,
1991), p. 331.

[2] G. Galli, R. M. Martin, R. Car, and M. Parrinello, Phys. Rev. Lett. 62, 555 (1989); Phys. Rev. B 42, 7470 (1990).

[3] J. Tersoff, Phys. Rev. Lett. 61, 2879 (1988).

[4] J. Tersoff, Phys. Rev. B 44, 12039 (1991).

[5] H.-P. Kaukonen and R. M. Nieminen, Phys. Rev. Lett. 68, 620 (1992)

[6] P. C. Kelires, Phys. Rev. Lett. 68, 1854 (1992).

[7] F. Li and J. S. Lannin, Phys. Rev. Lett. 65, 1905 (1990).

[8] D. R. McKenzie, D. Muller, and B. A. Pailthorpe, Phys. Rev. Lett. 67, 773 (1991).

[9] P. H. Gaskell, A. Saeed, P. Chieux, and D. R. McKenzie, Phys. Rev. Lett. 67, 1286 (1991).

[10] H. Pan, M. Pruski, B. C. Gerstein, F. Li, and J. S. Lannin, Phys. Rev. B 44, 6741 (1991).

[11] G. Comelli, J. Stohr, C. J. Robinson, and W. Jark, Phys. Rev. B 38, 7511 (1988).

[12] C. H. Xu, C. Z. Wang, C. T. Chan, and K. M. Ho, J. Phys. Condens. Matter 4, 6047 (1992).

[13] C. Z. Wang, C. H. Xu, B. L. Zhang, C. T. Chan, and K. M. Ho, in Physics and Chemistry of Finite Systems: From Cluster to Crystals, edited by P. Jena, S. N. Khanna, and B. K. Rao (Kluwer Academic, Boston/London, 1992), p. 1391.

[14] B. L. Zhang, C. Z. Wang, and K. M. Ho, Chem. Phys. Lett. 193, 225 (1992).

[15] For each density, the liquid configuration is generated by compressing a liquid structure of density of $2.0 \mathrm{~g} / \mathrm{cm}^{3}$ at $T=5000 \mathrm{~K}$ previously prepared by TBMD using more than 10 ps of simulation time. After the compression, the new liquid structure is allowed to equilibrate over 2000 MD steps (corresponding to $2 \mathrm{ps}$ ).

[16] H. C. Andersen, J. Chem. Phys. 72, 2384 (1980).

[17] We use the algorithm proposed by D. S. Franzblau, Phys. Rev. B 44, 4925 (1991).

[18] J. Robertson and E. P. O'Reilly, Phys. Rev. B 35, 2946 (1987).

[19] C. T. Chan (unpublished).

[20] Z. Zhu, C. T. Chan, and K. M. Ho (unpublished).

[21] D. Beeman, J. Silverman, R. Lynds, and M. R. Anderson, Phys. Rev. B 30, 870 (1984).

[22] D. Wesner et al., Phys. Rev. B 28, 2152 (1983). 


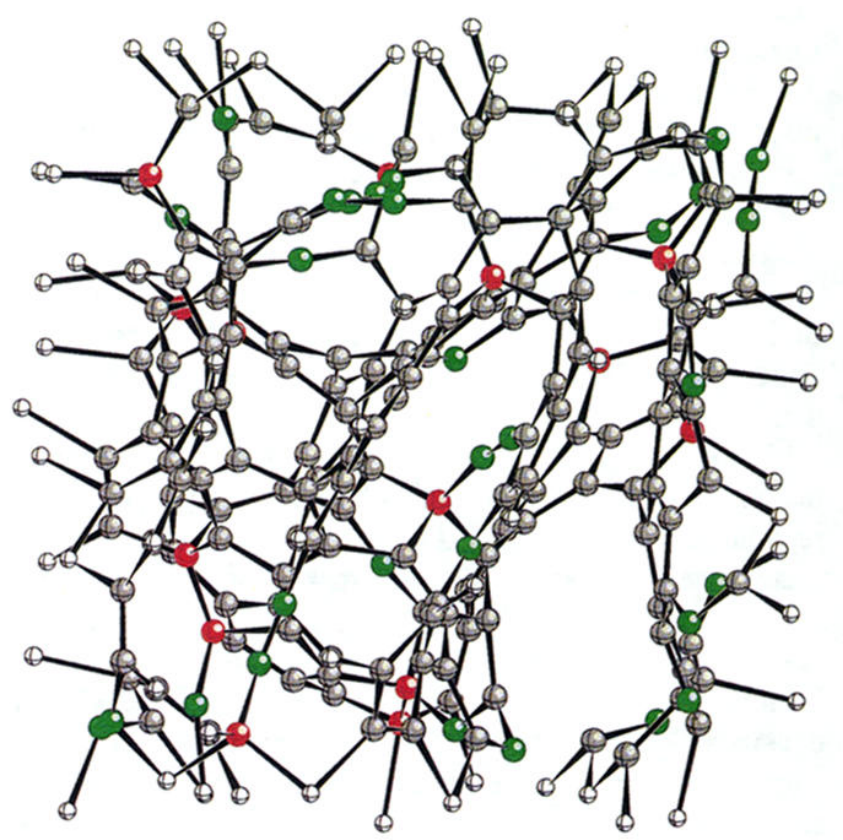

FIG. 2. Microscopic structure of the TBMD-generated $a-C$ network. The density of the sample is $2.2 \mathrm{~g} / \mathrm{cm}^{3}$. Various coordinated atoms are colored differently (twofold: green; threefold: black and white; fourfold: red). Smaller balls represent the carbon atoms outside the central cell (periodic boundary conditions are used). 